\title{
Central limit theorem and related results for the elephant random walk
}

Cristian F. Coletti, Renato Gava, and Gunter M. Schütz

Citation: Journal of Mathematical Physics 58, 053303 (2017);

View online: https://doi.org/10.1063/1.4983566

View Table of Contents: http://aip.scitation.org/toc/jmp/58/5

Published by the American Institute of Physics

\section{Articles you may be interested in}

Fisher information and topological pressure

Journal of Mathematical Physics 58, 052702 (2017); 10.1063/1.4983661

Phase space distribution of Riemann zeros

Journal of Mathematical Physics 58, 053504 (2017); 10.1063/1.4982737

Linear stochastic Schrödinger equations in terms of quantum Bernoulli noises

Journal of Mathematical Physics 58, 053510 (2017); 10.1063/1.4983660

Generations of solvable discrete-time dynamical systems

Journal of Mathematical Physics 58, 052701 (2017); 10.1063/1.4982959

Variable-length analog of Stavskaya process: A new example of misleading simulation Journal of Mathematical Physics 58, 053304 (2017); 10.1063/1.4983567

Circumspheres of sets of $\mathrm{n}+1$ random points in the d-dimensional Euclidean unit ball $(1 \leq \mathrm{n} \leq \mathrm{d})$ Journal of Mathematical Physics 58, 053301 (2017); 10.1063/1.4982640

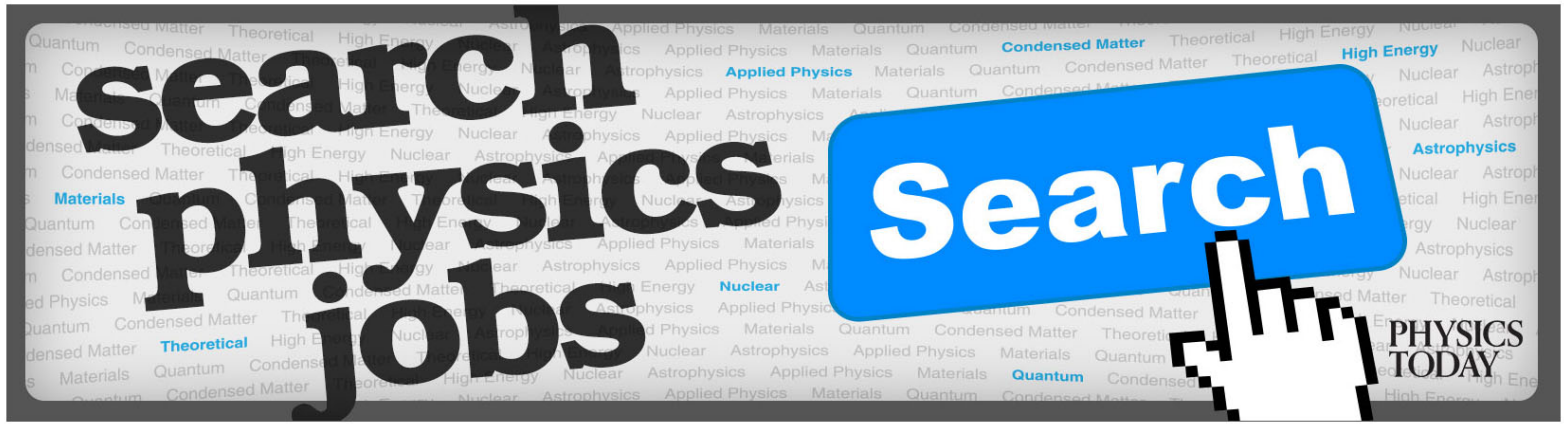




\title{
Central limit theorem and related results for the elephant random walk
}

\author{
Cristian F. Coletti, ${ }^{1, a)}$ Renato Gava, ${ }^{2, b)}$ and Gunter M. Schütz ${ }^{3, c)}$ \\ ${ }^{1}$ Centro de Matemática, Computação e Cognição, Universidade Federal do ABC, \\ Avenida dos Estados, 5001 Santo André, São Paulo, Brazil \\ ${ }^{2}$ Departamento de Estatística, Universidade Federal de São Carlos, Rodovia Washington Luiz, \\ Km 235, 13565-905 São Carlos, São Paulo, Brazil \\ ${ }^{3}$ Institute of Complex Systems II, Forschungszentrum Jülich, 52425 Jülich, Germany
}

(Received 2 September 2016; accepted 3 May 2017; published online 19 May 2017)

\begin{abstract}
We study the so-called elephant random walk (ERW) which is a non-Markovian discrete-time random walk on $\mathbb{Z}$ with unbounded memory which exhibits a phase transition from a diffusive to superdiffusive behavior. We prove a law of large numbers and a central limit theorem. Remarkably the central limit theorem applies not only to the diffusive regime but also to the phase transition point which is superdiffusive. Inside the superdiffusive regime, the ERW converges to a non-degenerate random variable which is not normal. We also obtain explicit expressions for the correlations of increments of the ERW. Published by AIP Publishing. [http://dx.doi.org/10.1063/1.4983566]
\end{abstract}

\section{INTRODUCTION}

In this paper, we consider a model presented in Ref. 9, the so-called elephant random walk (ERW). This is a discrete-time random walk $X_{n}$ on $\mathbb{Z}$ with unbounded memory whose random increments at each time step depend on the whole history of the process. This model is a rare example of a nonMarkovian process for which exact results on the moments of $X_{n}$ are available. ${ }^{2,8,9}$ Also some large deviation results have been obtained. ${ }^{5}$ Significantly, there is a phase transition from a diffusive to superdiffusive behavior as a function of a memory parameter $p$ (defined below) at a critical value $p_{c}$. A modified ERW was shown to exhibit also subdiffusion. ${ }^{6}$ Recently a surprising connection between the ERW and bond percolation on random recursive trees has been found. ${ }^{7}$

An open question that has remained is the actual probability distribution of $X_{n}$ in the original ERW. Initially it was suggested that on large scales the probability density is Gaussian, obeying the Fokker-Planck equation with the time-dependent drift term. ${ }^{9}$ Later it was conjectured that the distribution is Gaussian in the diffusive regime, but not in the superdiffusive regime. ${ }^{2,8}$ Here we address this problem. We prove rigorously a law of large numbers (Theorem 1) for any non-trivial value of the memory parameter and a central limit theorem (Theorem 2) that is valid both in the diffusive regime and at the critical value $p_{c}$, where the ERW is already superdiffusive. Inside the superdiffusive regime, $X_{n}$ converges to a non-degenerate but non-normal random variable (Theorem 3 ). Some further insight is obtained by a precise result on the correlations between the random increments (Theorem 4).

The approach we use to study the limiting behavior of the ERW involves martingale techniques ${ }^{4,10}$ and may be related to the study of limit theorems for a class of correlated Bernoulli processes. We refer the reader interested in this topic to the work of Wu et al. ${ }^{11}$ and references therein. In this context, it is worth mentioning the recent work of González-Navarrete and Lambert ${ }^{3}$ where the authors propose an approach to construct and study Bernoulli sequences combining dependence and independence periods.

\footnotetext{
a)cristian.coletti@ufabc.edu.br

b) gava@ufscar.br

c) g.schuetz@fz-juelich.de
} 
The paper is organized as follows. In Sec. II, we define the process and present our main results which are proved in Section III. In Subsection III A, we derive some auxiliary results that are used in the proofs of Subsection III B. We mention that upon completion of this work related results on the ERW by Baur and Bertoin appeared in arXiv (meanwhile published in Phys. Rev. $\mathrm{E}^{1}$ ) where the authors prove similar results, using a mapping between the ERW and an urn model.

\section{DEFINITION OF THE ERW AND MAIN RESULTS}

The ERW is defined as follows. The walk starts at a specific point $X_{0}$ at time $n=0$. At each discrete time step, the elephant moves one step to the right or to the left, respectively, so

$$
X_{n+1}=X_{n}+\eta_{n+1},
$$

where $\eta_{n+1}= \pm 1$ is a random variable. The memory consists of the set of random variables $\eta_{n^{\prime}}$ at previous time steps, which the elephant remembers as follows:

$\left(D_{1}\right)$ At time $n+1$, a number $n^{\prime}$ from the set $\{1,2, \ldots, n\}$ is chosen at random with probability $1 / n$.

$\left(D_{2}\right) \quad \eta_{n+1}$ is determined stochastically by the rule

$$
\eta_{n+1}=\eta_{n^{\prime}} \text { with probability } p \text { and } \eta_{n+1}=-\eta_{n^{\prime}} \text { with probability } 1-p \text {. }
$$

$\left(D_{3}\right)$ The elephant starting at $X_{0}$ moves to the right with probability $q$ and to the left with probability $1-q$, i.e.,

$$
\eta_{1}=1 \text { with probability } q \text { and } \eta_{1}=-1 \text { with probability } 1-q .
$$

It is obvious from the definition that

$$
X_{n}=X_{0}+\sum_{k=1}^{n} \eta_{k}
$$

From now on we consider $X_{0}=0$. Therefore, $X_{n}=\sum_{k=1}^{n} \eta_{k}$. A simple computation, using the total probability law, yields

$$
\mathbb{P}\left[\eta_{n+1}=\eta \mid \eta_{1}, \ldots, \eta_{n}\right]=\frac{1}{2 n} \sum_{k=1}^{n}\left[1+(2 p-1) \eta_{k} \eta\right] \text { for } n \geq 1,
$$

where $\eta= \pm 1$ (see Ref. 9 for details). For $n=0$, we get in accordance with rule $\left(D_{3}\right)$,

$$
\mathbb{P}\left[\eta_{1}=\eta\right]=\frac{1}{2}[1+(2 q-1) \eta]
$$

and

$$
\mathbb{E}\left[\eta_{1}\right]=2 q-1 .
$$

The conditional expectation of the increment $\eta_{n+1}$ given its previous history is given by

$$
\mathbb{E}\left[\eta_{n+1} \mid \eta_{1}, \ldots, \eta_{n}\right]=(2 p-1) \frac{X_{n}}{n} \text { for } n \geq 2 .
$$

In Ref. 9, Schütz and Trimper showed that

$$
\mathbb{E}\left[X_{n}\right]=(2 q-1) \frac{\Gamma(n+(2 p-1))}{\Gamma(2 p) \Gamma(n)} \sim \frac{2 q-1}{\Gamma(2 p)} n^{2 p-1} \text { for } n \gg 1 .
$$

Therefore,

$$
\mathbb{E}\left[\eta_{n+1}\right] \sim \frac{(2 p-1)(2 q-1)}{\Gamma(2 p)} n^{2(p-1)} .
$$

Now we state the main results. 
Theorem 1. Let $\left(X_{n}\right)_{n \geq 1}$ be the elephant random walk. Then

$$
\lim _{n \rightarrow \infty} \frac{X_{n}-\mathbb{E}\left[X_{n}\right]}{n}=0 \text { a.s. }
$$

for any value of $q$ and $p \in[0,1)$.

Remark 1. If $p=1$, which is not covered by the law of large numbers of Theorem 1, the ERW is trivial since by definition of the process one then has $\eta_{n}=\eta_{1}$ for all $n \geq 1$. Hence $X_{n} / n=\eta_{1}$ reduces to a binary random variable.

Interestingly, as the next two theorems demonstrate, the non-Markovian nature of the ERW is somewhat disguised for $p \leq 3 / 4$, but shows up for $p>3 / 4$.

Theorem 2. Let $\left(X_{n}\right)_{n \geq 1}$ be the elephant random walk and let $p \leq 3 / 4$.

(a) If $p<3 / 4$, then

$$
\frac{X_{n}-\frac{2 q-1}{\Gamma(2 p)} n^{2 p-1}}{\sqrt{\frac{n}{3-4 p}}} \stackrel{\mathcal{D}}{\rightarrow} \mathcal{N}(0,1) .
$$

(b) If $p=3 / 4$, then

$$
\frac{X_{n}-\frac{2 q-1}{\Gamma(3 / 2)} n^{1 / 2}}{\sqrt{n \ln n}} \stackrel{\mathcal{D}}{\longrightarrow} \mathcal{N}(0,1) .
$$

Theorem 3. Let $\left(X_{n}\right)_{n \geq 1}$ be the elephant random walk. If $3 / 4<p \leq 1$, then

$$
\frac{X_{n}}{n^{2 p-1} \Gamma(2 p)^{-1}}-(2 q-1) \rightarrow \text { M a.s. }
$$

where $M$ is a non-degenerate mean-zero random variable which is not normally distributed.

The fact that, for $p>3 / 4$, the rescaled elephant random walk does not converge in distribution to a random variable with normal distribution suggests that cross-correlations between the increments $\eta_{n}$ are in some sense "too strong." The following theorem quantifies these correlations.

Theorem 4. Let $\left(X_{n}\right)_{n \geq 1}$ be the elephant random walk with $q=1 / 2$ and $0 \leq p \leq 1$ and define

$$
F(n):=\frac{2 p-1}{3-4 p}\left(\frac{2(1-p)}{n}-\frac{(2 p-1) \Gamma(n+4 p-3)}{\Gamma(4 p-2) n !}\right) .
$$

Then, $\mathbb{E}\left[\eta_{n}\right]=0$ and

$$
\mathbb{E}\left[\eta_{n} \eta_{n+k}\right]=\frac{n ! \Gamma(2 p+n+k-2)}{(n+k-1) ! \Gamma(2 p-1+n)} F(n)
$$

for all $n \geq 1$ and $k \geq 1$.

Remark 2. For $p=3 / 4$ one has

$$
F(n)=\frac{1}{2 n}\left(1+\frac{1}{2} \sum_{m=1}^{n-1} \frac{1}{m}\right) .
$$

For $k, n \rightarrow \infty$ with $x:=k / n$ fixed, the correlation function has the form

$$
\mathbb{E}\left[\eta_{n} \eta_{n+k}\right] \sim(1+x)^{-2(1-p)} F(n),
$$


where $F(n)$ decays algebraically except at the transition point $p=3 / 4$. In the various regimes, Stirling's formula for the Gamma-function gives

$$
F(n) \sim\left\{\begin{array}{lr}
\frac{2(2 p-1)(1-p)}{3-4 p} n^{-1} & p<3 / 4 \\
\frac{\ln n}{4 n} & p=3 / 4 \\
\frac{(2 p-1)^{2}}{(4 p-3) \Gamma(4 p-2)} n^{-4(1-p)} & p>3 / 4 .
\end{array}\right.
$$

It appears that the transition at $p=3 / 4$ is driven by the strength of the correlations at time step $n$ rather than by their decay with the time-lag $k$ between increments.

\section{PROOFS}

In order to prove the limit theorems for the ERW stated in Section II, we use results from martingale theory proved in Refs. 3 and 10. The ideas employed here to prove Theorems 1 and 2 can also be found in the work of Wu et al. ${ }^{11}$ in a different context.

\section{A. Auxiliary results}

In this subsection, we present some results that will be used in Subsection III B where we prove Theorems 1-4.

Put

$$
a_{1}=1 \text { and } a_{n}=\prod_{j=1}^{n-1}\left(1+\frac{(2 p-1)}{j}\right) \text { for } n \geq 2 .
$$

Define the filtration $\mathcal{F}_{n}=\sigma\left(\eta_{1}, \ldots, \eta_{n}\right)$ and $M_{n}=\frac{X_{n}-\mathbb{E}\left[X_{n}\right]}{a_{n}}$ for $n \geq 1$. We claim that $\left\{M_{n}\right\}_{n \geq 1}$ is a martingale with respect to $\left\{\mathcal{F}_{n}\right\}_{n \geq 1}$, for

$$
\begin{aligned}
\mathbb{E}\left[M_{n+1} \mid \mathcal{F}_{n}\right] & =\frac{\left(X_{n}-\mathbb{E}\left[X_{n}\right]\right)}{a_{n+1}}+\frac{\mathbb{E}\left[\eta_{n+1} \mid \mathcal{F}_{n}\right]-\mathbb{E}\left[\eta_{n+1}\right]}{a_{n+1}} \\
& =\frac{\left(X_{n}-\mathbb{E}\left[X_{n}\right]\right)}{a_{n+1}}+\frac{(2 p-1) \frac{X_{n}}{n}-(2 p-1) \frac{\mathbb{E}\left[X_{n}\right]}{n}}{a_{n+1}} \\
& =\frac{\left(X_{n}-\mathbb{E}\left[X_{n}\right]\right)}{a_{n+1}}+\frac{\frac{(2 p-1)}{n}\left(X_{n}-\mathbb{E}\left[X_{n}\right]\right)}{a_{n+1}} \\
& =\left(X_{n}-\mathbb{E}\left[X_{n}\right]\right) \frac{\left(1+\frac{(2 p-1)}{n}\right)}{a_{n+1}} \\
& =M_{n} .
\end{aligned}
$$

Before proving the next lemma, let us make an important remark on $a_{n}$. Using the gamma function, we can rewrite $a_{n}$ in the following way:

$$
a_{n}=\frac{\Gamma(n+2 p-1)}{\Gamma(n) \Gamma(2 p)} \sim \frac{n^{2 p-1}}{\Gamma(2 p)} \text { as } n \rightarrow \infty .
$$

Notice that $a_{n} \rightarrow \infty$ as $n \rightarrow \infty$ if $p>1 / 2, a_{n}=1$ for $n \geq 1$ if $p=1 / 2$, and $a_{n} \rightarrow 0$ if $p<1 / 2$.

Lemma 1. The series $\sum_{n=1}^{\infty} \frac{1}{a_{n}^{2}}$ converges if and only if $p>3 / 4$.

Proof. Let $b_{n}=\frac{1}{a_{n}^{2}}$ and assume that $p \neq 3 / 4$. Then,

$$
n\left(1-\frac{b_{n}}{b_{n+1}}\right)=\frac{2(2 p-1)+\frac{(2 p-1)^{2}}{n}}{1+2 \frac{(2 p-1)}{n}+\frac{(2 p-1)^{2}}{n^{2}}} .
$$


Therefore,

$$
R:=\lim _{n \rightarrow+\infty} n\left(1-\frac{b_{n}}{b_{n+1}}\right)=4 p-2 .
$$

By the Raabe criteria, the series is convergent if $R>1$ and it is divergent if $R<1$. Note that $R>1$ if and only if $p>\frac{3}{4}$ and $R<1$ if and only if $p<\frac{3}{4}$.

Assume now that $p=\frac{3}{4}$. Since $2 p-1=1 / 2>0$, it follows by (5) that

$$
a_{n}=\prod_{j=1}^{n-1}\left(1+\frac{1 / 2}{j}\right) \sim \frac{n^{1 / 2}}{\sqrt{\pi / 4}} .
$$

Thence $1 / a_{n}^{2} \geq \frac{1}{2 n}$ for $n$ large enough and $\sum_{n=2}^{\infty} \frac{1}{a_{n}^{2}}$ diverges.

Let $\left(D_{n}\right)_{n \geq 1}$ be the martingale differences defined by $D_{1}=M_{1}$ and, for $n \geq 2, D_{n}=M_{n}-M_{n-1}$. Observe that

$$
\begin{aligned}
D_{j} & =\frac{X_{j}-\mathbb{E}\left[X_{j}\right]}{a_{j}}-\frac{X_{j-1}-\mathbb{E}\left[X_{j-1}\right]}{a_{j-1}} \\
& =\frac{\eta_{j}-\mathbb{E}\left[\eta_{j}\right]}{a_{j}}-\frac{\left(X_{j-1}-\mathbb{E}\left[X_{j-1}\right]\right)}{j-1} \frac{2 p-1}{a_{j}} .
\end{aligned}
$$

Furthermore, since the increments $\eta_{j}$ 's are uniformly bounded, it is trivial to see that

$$
\left|D_{j}\right| \leq \frac{4}{a_{j}}
$$

We state without proof the Kronecker lemma.

Lemma 2. Let $\left(x_{n}\right)_{n \geq 1}$ and $\left(b_{n}\right)_{n \geq 1}$ be sequences of real numbers such that $0<b_{n} \nearrow+\infty$. If $\sum_{k} x_{k} / b_{k}$ converges, then $\lim _{n \rightarrow+\infty} \frac{\sum_{k=1}^{n} x_{k}}{b_{n}}=0$.

\section{B. Proofs of the main results}

\section{Strong law of large numbers}

Proof of Theorem 1. First observe that

$$
\frac{a_{n}}{n}=\frac{1}{n} \prod_{j=1}^{n-1}\left(1+\frac{2 p-1}{j}\right)=\prod_{j=1}^{n-1} \frac{j+2 p-1}{j+1}=\prod_{j=1}^{n-1}\left(1-\frac{2(1-p)}{j+1}\right),
$$

where $0 \leq \frac{j+2 p-1}{j+1} \leq 1$. Observe that $\sum_{j=1}^{\infty} \frac{2(1-p)}{j+1}=\infty$ implies that $\lim _{n \rightarrow+\infty} \frac{a_{n}}{n}=0$. Furthermore, it follows from (7) and the fact that $0 \leq \frac{j+2 p-1}{j+1} \leq 1$ that $\left(a_{n} / n\right)_{n \geq 1}$ is a non-increasing sequence.

Define $N_{j}=\frac{a_{j}}{j} D_{j}$. By (6), we may conclude that $\left(N_{j}\right)_{j \geq 1}$ is a sequence of martingale differences such that

$$
\sum_{j=1}^{+\infty} \mathbb{E}\left[N_{j}^{2} \mid \mathcal{F}_{j-1}\right] \leq \sum_{j=1}^{+\infty} \frac{4^{2}}{j^{2}}<+\infty \text { a.s. }
$$

Now, Theorem 2.17 in Ref. 3 implies that $\sum_{j=1}^{+\infty} N_{j}$ converges a.s. Since $n / a_{n} \nearrow \infty$, a direct application of Lemma 2 gives

$$
\frac{a_{n}}{n} \sum_{j=1}^{n} D_{j}=\frac{a_{n}}{n} M_{n} \rightarrow 0 \text { a.s. }
$$


Next, note that

$$
\frac{X_{n}-\mathbb{E}\left[X_{n}\right]}{n}=\frac{a_{n}}{n} M_{n},
$$

i.e., the law of large numbers holds for $X_{n}$.

Corollary 1. Let $\left(X_{n}\right)_{n \geq 1}$ be the elephant random walk. Then

$$
\lim _{n \rightarrow \infty} \frac{X_{n}}{n}=0 \text { a.s. }
$$

for any value of $q$ and $p \in[0,1)$.

Proof. It follows from (3) that $\frac{\mathbb{E}\left[X_{n}\right]}{n} \sim \frac{2 q-1}{\Gamma(2 p)} n^{2(p-1)}$, which in turn goes to 0 as $n \rightarrow \infty$, and the claim follows.

\section{Central limit theorem}

Proof of Theorem 2. Define

$$
s_{1}^{2}=q(1-q) \text { and } s_{n}^{2}:=q(1-q)+\sum_{j=2}^{n} \frac{1}{a_{j}^{2}} \text { for } n \geq 2 .
$$

Before proving the results, we need to say that both claims in Theorem 2 amount to show that

$$
\frac{X_{n}-\mathbb{E}\left[X_{n}\right]}{a_{n} s_{n}} \stackrel{\mathcal{D}}{\longrightarrow} \mathcal{N}(0,1) .
$$

Indeed, we will show that (8) holds. First recall that $\mathbb{E}\left(X_{n}\right) \sim(2 q-1) n^{2 p-1} / \Gamma(2 p)$, by (3). Combining Lemma 1 and (5) we get

$$
\begin{aligned}
& s_{n}^{2} \sim \Gamma(2 p)^{2} \frac{n^{3-4 p}}{3-4 p}, \text { if } p<3 / 4, \\
& s_{n}^{2} \sim \Gamma(3 / 2)^{2} \ln n, \text { if } p=3 / 4,
\end{aligned}
$$

which in turn implies that $a_{n} s_{n} \sim \sqrt{n \ln n}$, if $p=3 / 4$, and $a_{n} s_{n} \sim \sqrt{n /(3-4 p)}$, if $p<3 / 4$.

We now turn our attention to the proof of (8). We will verify the two conditions of Corollary 3.1 of Ref. 3 in order to get our result.

We begin by checking the conditional Lindeberg condition. Let $D_{n j}=\frac{D_{j}}{s_{n}}$ for $1 \leq j \leq n$. Given $\varepsilon>0$, we need to prove that

$$
\sum_{j=1}^{n} \mathbb{E}\left(D_{n j}^{2} \mathbb{I}\left(\left|D_{n j}\right|>\varepsilon\right) \mid \mathcal{F}_{j-1}\right) \rightarrow 0 \text { a.s.. }
$$

If $p \in[1 / 2,3 / 4]$, then $a_{n} \geq 1$ and $s_{n} \rightarrow \infty$. Next note that $\left\{\left|D_{n j}\right|>\varepsilon\right\} \subset\left\{\frac{4}{s_{n}}>\varepsilon\right\}$, but the last set is empty for $n$ large enough, so (9) holds. If $p<1 / 2$, observe that $\lim _{n \rightarrow \infty} a_{n} s_{n}=\infty$ and $a_{j}^{-1} \leq a_{n}^{-1}$ for $j=1, \ldots, n$. Then it is easy to see that $\left\{\left|D_{n j}\right|>\varepsilon\right\} \subset\left\{\frac{4}{a_{n} s_{n}}>\varepsilon\right\}$, and again the latter set is empty for $n$ sufficiently large.

Next we check the conditional variance condition. Since $\frac{\sum_{j=1}^{n} D_{j}}{s_{n}}=\frac{S_{n}-\mathbb{E}\left[S_{n}\right]}{a_{n} s_{n}}$, we must estimate $\mathbb{E}\left[D_{j}^{2} \mid \mathcal{F}_{j-1}\right]$. Observe that

$$
\begin{aligned}
D_{j}^{2}= & \frac{1}{a_{j}^{2}}\left(\left(1-2 \mathbb{E}\left[\eta_{j}\right] \eta_{j}+\mathbb{E}^{2}\left[\eta_{j}\right]\right)+\left(\frac{\left(X_{j-1}-\mathbb{E}\left[X_{j-1}\right]\right)}{j-1}\right)^{2}(2 p-1)^{2}\right) \\
& -\frac{2}{a_{j}^{2}}\left(\left(\eta_{j}-\mathbb{E}\left[\eta_{j}\right]\right)\left(\frac{\left(X_{j-1}-\mathbb{E}\left[X_{j-1}\right]\right)}{j-1}\right)(2 p-1)\right),
\end{aligned}
$$


since $\eta_{j}^{2}=1$.

It follows from the law of large numbers for $X_{n}$ that

$$
\mathbb{E}\left[\frac{1}{a_{j}^{2}}\left(\frac{\left(X_{j-1}-\mathbb{E}\left[X_{j-1}\right]\right)}{j-1}\right)^{2}(2 p-1)^{2} \mid \mathcal{F}_{j-1}\right]
$$

and

$$
\mathbb{E}\left[\frac{1}{a_{j}^{2}}\left(\left(\eta_{j}-\mathbb{E}\left[\eta_{j}\right]\right)\left(\frac{\left(X_{j-1}-\mathbb{E}\left[X_{j-1}\right]\right)}{j-1}\right)(2 p-1)\right) \mid \mathcal{F}_{j-1}\right]
$$

are o $\left(\frac{1}{a_{j}^{2}}\right)$. Therefore, in order to obtain the asymptotic behavior of $\mathbb{E}\left[D_{j}^{2} \mid \mathcal{F}_{j-1}\right]$, we need to study the behavior of $\frac{1}{a_{j}^{2}}\left(\mathbb{E}\left[\left(1-2 \mathbb{E}\left[\eta_{j}\right] \eta_{j}+\mathbb{E}^{2}\left[\eta_{j}\right]\right) \mid \mathcal{F}_{j-1}\right]\right)$.

It follows from (1) and (4) that, for $j \geq 2$,

$$
\begin{aligned}
\frac{1}{a_{j}^{2}} \mathbb{E}\left[\left(1-2 \mathbb{E}\left[\eta_{j}\right] \eta_{j}+\mathbb{E}^{2}\left[\eta_{j}\right]\right) \mid \mathcal{F}_{j-1}\right] & \sim \frac{1}{a_{j}^{2}}\left(1-2 \frac{(2 p-1)(2 q-1)}{\Gamma(2 p)(j-1)^{2(1-p)}} \frac{(2 p-1) X_{j-1}}{j-1}\right) \\
& +\frac{1}{a_{j}^{2}}\left(\frac{(2 p-1)(2 q-1)}{\Gamma(2 p)(j-1)^{2(1-p)}}\right)^{2} \\
& =\frac{1}{a_{j}^{2}}+\mathrm{o}\left(\frac{1}{a_{j}^{2}}\right) .
\end{aligned}
$$

Therefore, for $j \geq 2$ we have that

$$
\mathbb{E}\left[D_{j}^{2} \mid \mathcal{F}_{j-1}\right]=\frac{1}{a_{j}^{2}}+\mathrm{o}\left(\frac{1}{a_{j}^{2}}\right) .
$$

Finally, for $j=1$, we have that

$$
\mathbb{E}\left[D_{1}^{2} \mid \mathcal{F}_{0}\right]=\frac{1}{a_{1}^{2}} q(1-q),
$$

where $a_{1}=1$. Recall that $s_{1}^{2}=q(1-q)$ and $s_{n}^{2}:=q(1-q)+\sum_{j=2}^{n} \frac{1}{a_{j}^{2}}$ for $n \geq 2$. From Lemma 1 , we have that $s_{n} \rightarrow \infty$ as $n \rightarrow \infty$ if and only if $p \leq 3 / 4$. From this fact, (10), (11), and Theorem 1, we may conclude that

$$
\frac{1}{s_{n}^{2}} \sum_{j=1}^{n} \mathbb{E}\left[D_{j}^{2} \mid \mathcal{F}_{j-1}\right] \stackrel{a . s}{\longrightarrow} 1 .
$$

Therefore, by Corollary 3.1 in Ref. 3 we conclude that

$$
\sum_{j=1}^{n} D_{n j}=\frac{X_{n}-\mathbb{E}\left[X_{n}\right]}{a_{n} s_{n}} \stackrel{\mathcal{D}}{\longrightarrow} \mathcal{N}(0,1) .
$$

\section{Almost sure convergence for $p>\frac{3}{4}$}

Proof of Theorem 3. We begin with an elementary observation. It follows from (3) and (5) that $\mathbb{E}\left(X_{n}\right)=(2 q-1) a_{n}$, where $a_{n} \sim n^{2 p-1} / \Gamma(2 p)$. Lemma 1 says that if $p>\frac{3}{4}$, then $\sum_{n=1}^{\infty} \frac{1}{a_{n}^{2}}<\infty$. Since $\left|D_{j}\right| \leq \frac{4}{a_{j}}$ (see (6)), then Theorem 12.1 in Ref. 10 implies that $M_{n}=\sum_{j=1}^{n} D_{j}=\frac{X_{n}-\mathbb{E}\left[X_{n}\right]}{a_{n}} \rightarrow M$ a.s. and in $\mathcal{L}^{2}$. These results and $\mathbb{E}\left(M_{n}\right)=0$ together imply that

$$
|\mathbb{E}(M)|=\left|\mathbb{E}\left(M-M_{n}\right)\right| \leq \mathbb{E}\left(\left|M-M_{n}\right|\right) \leq \mathbb{E}\left(\left|M-M_{n}\right|^{2}\right)^{1 / 2} \rightarrow 0 \text { as } n \rightarrow \infty .
$$


Furthermore, the fact that $\left(M_{n}\right)_{n \geq 1}$ is a bounded martingale in $\mathcal{L}^{2}$ plus its a.s. convergence to $M$ imply that

$$
\operatorname{Var}(M)=\lim _{n \rightarrow \infty} \operatorname{Var}\left(M_{n}\right)=\sum_{j=1}^{\infty} \mathbb{E}\left(D_{j}^{2}\right)>0 .
$$

Then, $M$ is not a degenerate random variable. The claim that $M$ is not normal follows from the explicit form of the skewness and kurtosis of the distribution of $X_{n}$ obtained by exact computation in Ref. 8.

\section{E. Correlations}

Proof of Theorem 4. To compute the correlation function $\mathbb{E}\left[\eta_{n+k} \eta_{n}\right]$ for $n \geq 1$ and $k \geq 1$, we introduce the auxiliary quantity $H_{k}=(n+k-1) \mathbb{E}\left[\eta_{n+k} X_{n}\right]$. It follows from (2) that

$$
H_{k+1}=\left(1+\frac{2 p-1}{n+k-1}\right) H_{k}, \quad H_{1}=(2 p-1) \mathbb{E}\left[X_{n}^{2}\right],
$$

which yields by induction

$$
H_{k}=\frac{(n-1) ! \Gamma(2 p+n+k-2)}{(n+k-2) ! \Gamma(2 p-1+n)} H_{1}
$$

Therefore,

$$
\begin{aligned}
\mathbb{E}\left[\eta_{n+k} X_{n}\right] & =\frac{n ! \Gamma(2 p+n+k-2)}{(n+k-1) ! \Gamma(2 p-1+n)} \mathbb{E}\left[\eta_{n+1} X_{n}\right] \\
& =(2 p-1) \frac{(n-1) ! \Gamma(2 p+n+k-2)}{(n+k-1) ! \Gamma(2 p-1+n)} \mathbb{E}\left[X_{n}^{2}\right] .
\end{aligned}
$$

Subtracting $\mathbb{E}\left[\eta_{n+k} X_{n-1}\right]$ yields $\mathbb{E}\left[\eta_{n+k} \eta_{n}\right]$ in the form stated in the theorem with

$$
F(n)=\frac{2 p-1}{n}\left[\mathbb{E}\left[X_{n}^{2}\right]-\left(1+\frac{2 p-1}{n-1}\right) \mathbb{E}\left[X_{n-1}^{2}\right]\right] .
$$

With the explicitly known expression ${ }^{9}$

$$
\mathbb{E}\left[X_{n}^{2}\right]=\frac{n}{4 p-3}\left(\frac{\Gamma(n+4 p-2)}{\Gamma(4 p-2) \Gamma(n+1)}-1\right),
$$

we arrive at the desired result.

\section{ACKNOWLEDGMENTS}

C.F.C. thanks FAPESP (Grant No. 2015/20110-0) for financial support. C.F.C. was visiting Istituto Politecnico di Milano when part of this work was carried out and he is grateful for their hospitality and support. R.G. thanks CNPq (Grant No. 461365/2014-6) for financial support. G.M.S. thanks the University of São Paulo for kind hospitality.

${ }^{1}$ Baur, E. and Bertoin, J., "Elephant random walks and their connection to Pólya-type urns,” Phys. Rev. E 94, 052134 (2016).

2 da Silva, M. A. A., Cressoni, J. C., Schütz, G. M., Viswanathan, G. M., and Trimper, S., "Non-Gaussian propagator for elephant random walks," Phys. Rev. E 88, 022115 (2013).

${ }^{3}$ González-Navarrete, M. and Lambert, R., "On dependent Bernoulli sequences with memory lapses," e-print arXiv:1607.08299.

${ }^{4}$ Hall, P. and Heyde, C. C., Martingale Limit Theory and its Application (Academic Press, New York, 1980).

${ }^{5}$ Harris, R. J. and Touchette, H., "Current fluctuations in stochastic systems with long-range memory," J. Phys. A: Math. Theor. 42, 342001 (2009).

${ }^{6}$ Kumar, N., Harbola, U., and Lindenberg, K., "Memory-induced anomalous dynamics: Emergence of diffusion, subdiffusion, and superdiffusion from a single random walk model," Phys. Rev. E 82, 021101 (2010).

${ }^{7}$ Kürsten, R., "Random recursive trees and the elephant random walk," Phys. Rev. E 93, 032111 (2016).

${ }^{8}$ Paraan, F. N. C. and Esguerra, J. P., "Exact moments in a continuous time random walk with complete memory of its history," Phys. Rev. E 74, 032101 (2006).

${ }^{9}$ Schütz, G. M. and Trimper, S., "Elephants can always remember: Exact long-range memory effects in a non-Markovian random walk," Phys. Rev. E 70, 045101 (2004).

${ }^{10}$ Williams, D., Probability with Martingales (Cambridge University Press, Cambridge, 1991).

${ }^{11}$ Wu, L., Qi, Y., and Yang, J., “Asymptotics for dependent Bernoulli random variables,” Statist. Probab. Lett. 82(3), 455-463 (2012). 